\title{
On the reaction time of some synchronous systems
}

\author{
Ilias Garnier, Christophe Aussaguès, Vincent David \\ CEA, LIST, Embedded Real Time Systems Laboratory \\ Point Courrier 94, Gif-sur-Yvette, F-91191 France \\ Firstname. Lastname@cea.fr
}

\author{
Guy Vidal-Naquet \\ SUPELEC Systems Sciences (E3S) \\ Computer Science Department \\ 91192 Gif-sur-Yvette Cedex, France \\ Guy.Vidal-Naquetesupelec.fr
}

\begin{abstract}
This paper presents an investigation of the notion of reaction time in some synchronous systems. A state-based description of such systems is given, and the reaction time of such systems under some classic composition primitives is studied. Reaction time is shown to be non-compositional in general. Possible solutions are proposed, and applications to verification are discussed. This framework is illustrated by some examples issued from studies on real-time embedded systems.
\end{abstract}

\section{Introduction}

A primary concern when developing hard real-time embedded systems is to ensure the timeliness of computations. This kind of requirement is often expressed as a reaction time constraint, i.e. an upper bound on the time the system may take to process an input and produce the related output. When systems are composed of multiple communicating agents, this task may be difficult. In this paper, we propose a formalization of reaction time for a certain class of synchronous systems. We show that reaction time is a fine-grained notion of functional dependency, and we show that it is non-compositional. In order to solve this problem, we propose an approximate but compositional method to reason on functional dependency and reaction time.

Related work. The specification and verification of temporal properties traditionally relies on temporal logic [6] or related formalisms [2]. With these formalisms, the system designer gives a specification of some causality or quantitative property which is then verified by model-checking.

These methods are also applicable to the restricted class of synchronous systems [8]. The OASIS [5] system, which motivated this study, belongs to this class. A traditional compositional verification of synchronous systems using Moore machines [10] was given in [4]. Our formal framework to reason on reaction time was heavily inspired by the literature on information flow analysis [3] and on the categorytheoretic view of process algebras [1]. It is also similar to testing methods [11].

\section{Preliminaries}

\subsection{Case study: proving the reactivity of a simple system}

Let $S$ be a black box with two buttons $A$ and $B$ as inputs and the elements of any non-singleton set as outputs. In this example, we will assume that $S$ is deterministic. Our goal is to decide whether pressing $A$ has any observable effect on the system. A naive solution is to verify whether the new observable state is different from the previous one.

$$
\begin{array}{l|lll}
\text { Input } & S & \stackrel{A}{\rightarrow} & S_{A} \\
\text { Observable state } & o & & o^{\prime}
\end{array}
$$

Bliudze, S., Bruni, R., Carbone, M., Silva, A. (Eds.); ICE 2011

EPTCS 59, 2011, pp. 69-83 doi 10.4204/EPTCS.59.7 
If $o \neq o^{\prime}$, we may consider that the system seems to have answered to the pressing of $A$. There are two counter-arguments to this conclusion.

1. $S$ may have decided in advance to output $o^{\prime}$;

2. there is no reason for an observable consequence to occur immediately after pressing the button.

In order to obtain a correct solution, the main point to take into account is that the observable state is not only function of the inputs but also of the internal state. From now on, we will assume that we have two identical copies of $S$, and we will proceed to the experiment simultaneously with the button $A$ and the button $B$.

$$
\begin{array}{l|lll|lll}
\text { Input } & S & \stackrel{A}{\rightarrow} & S_{A} & S & \stackrel{B}{\rightarrow} & S_{B} \\
\text { Observable state } & o & & o^{\prime} & o & & o^{\prime \prime}
\end{array}
$$

If $o^{\prime} \neq o^{\prime \prime}$, we deduce that the system has distinguished between pressing the button $A$ and the button $B$. This experiment is thus strictly more informative than the previous one. In the other case, knowing that $o^{\prime}=o^{\prime \prime}$ is not enough for us to extract any information on the internal behavior of the system. Indeed, the second counter-argument advances that the observable reaction can occur after an arbitrary number of transitions. A solution is to iterate the experiment on $S_{A}$ and $S_{B}$ until observing a difference, but the observable state then becomes possibly correlated to the other choices $A$ or $B$ performed during the experiment. The choices must thus be identical for $S_{A}$ and $S_{B}$.

We can informally define reactivity by stating that if there exists a finite sequence of experiments (i.e. a word on $\{A, B\}$ ) allowing to distinguish the systems $S_{A}$ and $S_{B}$, then $S$ is reactive. In formal terms, this is equivalent to stating that $S_{A}$ and $S_{B}$ must be non-bisimilar. The reaction time is the maximum length of the minimal experiment allowing to prove non-bisimilarity.

\subsection{Notations and definitions}

Some definitions will be useful to our work. Let $\Sigma$ be a set. The set of finite words on $\Sigma$ is noted $\Sigma^{*}$, and the set of infinite words is noted $\Sigma^{\omega} \equiv \mathbb{N} \rightarrow \omega$. The set of finite and infinite output words is $\Sigma^{\infty}=\Sigma^{*} \cup \Sigma^{\omega}$. The length of a finite word $w$ will be noted $|w|$. For any word $w$ we will note prefix $(w, l e n)$ the prefix of $w$ of length len, and we also note $w[i]$ the $i$-th symbol of $w$, where $i \in[0 ;|w|-1]$.

The singleton set is $\mathbb{1}=\{\star\}$ (up to isomorphism), and the disjoint union of two sets $A$ and $B$ is noted $A+B$. The set of natural integers strictly inferior to $x$ is noted $\mathbb{N}^{<x}$.

\section{A formal model of synchronous systems}

We aim at giving a formal model of synchronous systems sufficiently expressive to encode languages such as Lustre [8] and PsyC [5]. Our formalism is an adaptation of Moore machines [10].

\subsection{The synchronous abstraction}

We will restrict ourselves to the set of systems which respect the synchronous mode of computation. In this model, the computation is divided in successive rounds. Each transition from a round to the next denotes the tick of a global logical clock. The observable state of a system is constant on each round, and changes only at the boundary between rounds. At each new round, the new internal state is a function of the current input and internal state (equivalently, the internal state is a function of the initial internal state and all previous inputs). The observable state is only function of the internal state. The following timeline shows an example of a deterministic synchronous computation involving three successive rounds. 


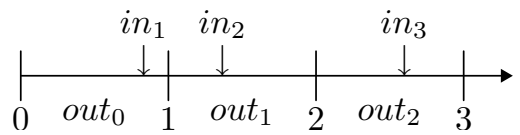

In this example, the output out $_{0}$ is a function of the initial internal state only; the output out $_{1}$ is a function of the initial internal state and $i n_{1}$; and the output out $t_{2}$ is a function of the initial internal state, $i n_{1}$ and $i n_{2}$. Thus, inputs have no immediate effect on the observable state.

\subsection{State-based description of synchronous systems}

We define our synchronous systems as a labeled transition system (LTS) inspired by Moore machines. We recall here some definitions which will be useful in the following developments.

Definition 1 (Synchronous system). Let In be a set of inputs and Out be a set of inputs. A synchronous system $S=\left\langle\right.$ In, Out $, Q, E$, out,$\left.q_{i}\right\rangle$ is the data of:

- a set of states $Q$,

- a transition relation $E \subseteq Q \times \operatorname{In} \times Q$,

- a labeling function associating states to outputs out: $Q \rightarrow$ Out,

- and an initial state $q_{i} \in Q$.

The sets In and Out are the signature of $S$. We will note $p \stackrel{a}{\rightarrow} q$ as a shorthand for $(p, a, q) \in E$. Moreover, we constrain our systems to be finitely branching and to be complete, i.e. $\forall p \in Q, \forall a \in \operatorname{In}, \exists p \stackrel{a}{\rightarrow} q{ }^{1}$

The computational meaning of a LTS is expressed using the notion of run. It allows to define the output language associated to an input word.

Definition 2 (Run of a synchronous system, output language). Let $S=\left\langle\right.$ In, Out, $Q, E$, out, $\left.q_{i}\right\rangle$ be a synchronous system. We define the notions of finite run and the associated output language.

Finite runs. Let $w \in I^{*}$ be a finite input word. The set of finite, maximal runs of $S$ on $w$ starting from state $q_{0} \in Q$ is $\operatorname{Runs}_{S}^{*}\left(q_{0}, w\right) \subseteq Q \times(\operatorname{In} \times Q)^{*}$ and is defined as:

$$
\operatorname{Runs}_{S}^{*}\left(q_{0}, w\right)=\left\{q_{0} \cdot w[0] \cdot q_{1} \ldots w[|w|-1] \cdot q_{|w|-1} \mid \forall i \in[0 ;|w|-1], q_{i} \stackrel{w[i]}{\longrightarrow} q_{i+1}\right\} .
$$

Output language. The output language of S associated to $w$ and $q_{0}$ is $\mathcal{L}_{S}^{*}\left(q_{0}, w\right) \subseteq$ Out ${ }^{*}$ and is defined as follows:

$$
\mathcal{L}_{S}^{*}\left(q_{0}, w\right)=\left\{\operatorname{out}\left(q_{0}\right) . \operatorname{out}\left(q_{1}\right) \ldots \operatorname{out}\left(q_{n-1}\right) \mid q_{0} \cdot a_{0} \cdot q_{1} \cdot a_{1} \ldots \in \operatorname{Runs_{S}^{*}}\left(q_{0}, w\right)\right\} .
$$

The classical equivalence relation on states of labeled transition systems is bisimilarity. Its definition is slightly adapted to our notion of synchronous system.

Definition 3 (Bisimilarity, non-bisimilarity). Let $S=\left\langle\right.$ In, Out, $Q, E$, out, $\left.q_{i}\right\rangle$ be a synchronous system. A relation $R \subseteq Q \times Q$ is said to be a (strong) bisimulation if and only if the following condition holds:

$$
\forall(p, q) \in R, \operatorname{out}(p)=\operatorname{out}(q) \wedge\left(\forall p \stackrel{a}{\rightarrow} p^{\prime}, \exists q \stackrel{a}{\rightarrow} q^{\prime},\left(p^{\prime}, q^{\prime}\right) \in R\right) \wedge\left(\forall q \stackrel{a}{\rightarrow} q^{\prime}, \exists p \stackrel{a}{\rightarrow} p^{\prime},\left(p^{\prime}, q^{\prime}\right) \in R\right) .
$$

If there exists such a relation $R$ s.t. $(p, q) \in R$, then $p$ and $q$ are said to be bisimilar, which is noted $p \sim q$. Moreover, bisimilarity is an equivalence relation. Conversely, the negation of bisimilarity $\nsim \subseteq Q \times Q$ is inductively defined by the rules below. In these rules, $p, q \in Q$ and $a \in$ In are universally quantified.

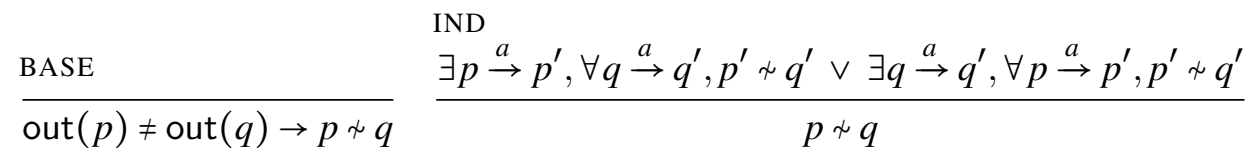

\footnotetext{
${ }^{1}$ In practice, these conditions constrain the input and output data sets to be finite.
} 


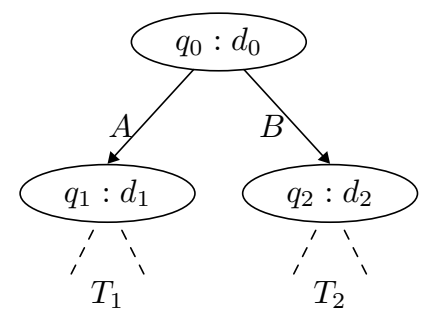

(a) Reactive deterministic system

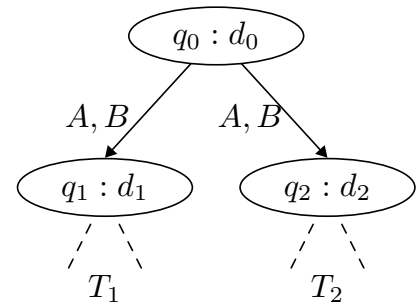

(b) Non-reactive non-det. sys.

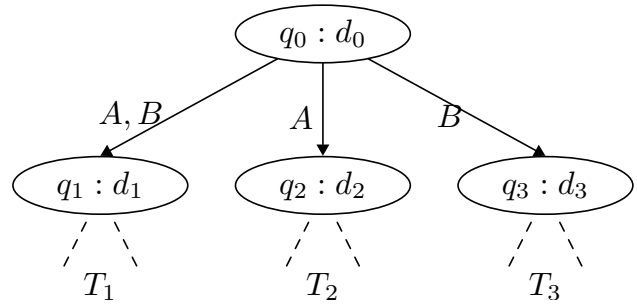

(c) Reactive non-deterministic system

Figure 1: Some reactive and non-reactive systems

In this paper, except when stated otherwise, all state spaces shall be assumed to be quotiented by bisimulation equivalence.

\section{Reaction time of a state in a synchronous system}

This section formalizes the ideas exposed in the case study, in Sec 2.1, and extends them to nondeterministic systems. The case study proposes to model reaction as a functional dependency between a set of inputs and the future behavior of the system. In our formal model, these future behaviors are represented as the successor states of the considered state.

In this setting, we will first define a notion of reactivity inspired by functional dependency, and then define reaction time as the necessary time to prove that two successor states are not bisimilar.

\subsection{Reactivity}

Let In $=\{A, B\}$ and Out be a non-singleton set. Let $S=\left\langle\right.$ In, Out, $Q$, out, $\left.E, q_{i}\right\rangle$ be a synchronous system. and let's assume that state $q \in Q$ is as depicted in Fig. 1(a). We observe that there are two inputs $A$ and $B$ leading to non-bisimilar states $q_{1}$ and $q_{2}$. In this case, state $q$ is thus reactive. In the case of non-deterministic systems, we must generalize this idea: if there exists an asymmetry in the possible transitions of a system, then it is reactive. Let's assume that state $q$ is as depicted in Fig. 1(b). There, $q$ is not reactive because there is a symmetry between the transitions possible with $A$ and the transitions possible with $B$. This symmetry is broken in Fig. 1(b), Non-determinism highlights the fact that reactivity is a kind of non-bisimilarity.

Definition 4 (Reactivity of a state in a synchronous system, separating pair). Let In, Out be two sets, $S=\left\langle\right.$ In, Out,$Q$, out, $\left.E, q_{i}\right\rangle$ be a synchronous system and $q \in Q$ be a state. We will denote reactive $(q)$ the fact that $q$ is reactive. The predicate reactive $(q)$ is defined as follows:

$$
\text { reactive }(q) \triangleq \exists a_{1}, a_{2} \in \operatorname{In}, a_{1} \neq a_{2} \wedge\left(\exists q \stackrel{a_{1}}{\rightarrow} q_{1}, \forall q \stackrel{a_{2}}{\rightarrow} q_{2}, q_{1} \nsim q_{2}\right) .
$$

The pair of inputs $\left(a_{1}, a_{2}\right)$ is a separating pair of $q$. It is deterministic iff $\forall q \stackrel{a_{1}}{\rightarrow} q_{1}, \forall q \stackrel{a_{2}}{\rightarrow} q_{2}, q_{1} \nsim q_{2}$. The set of separating pairs of $q$ is noted $\operatorname{SepPairs}(q)$, and the deterministic subset is $\operatorname{DSepPairs}(q)$.

\subsection{Observable effects}

Observable effects stem from a fine-grained study of reactivity. In this section, we show that an observable effect characterizes a temporally localized difference between the behaviors of non-bisimilar states. 


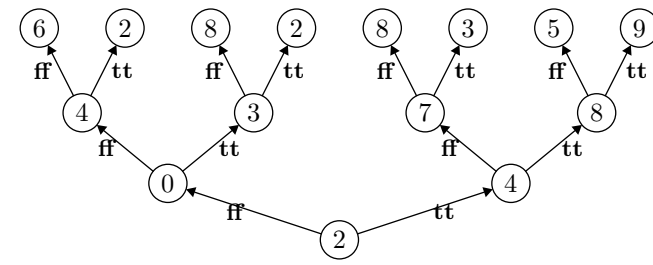

(a)

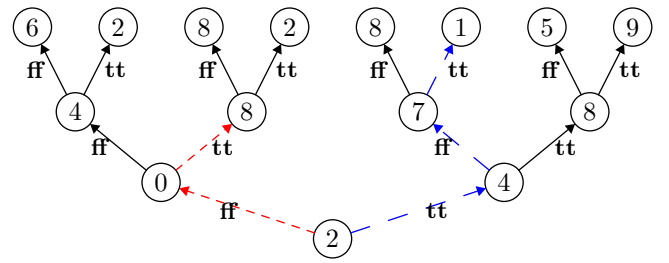

(b)

Figure 2: A pair of separable LTS

We show that an input data has observable effects on the system on a not necessarily finite interval.

Characterizing the difference between two states. Characterizing difference between states can be done by studying the negation of bisimulation.

Definition 5 (Separators, strongly separable states). Let $S=\left\langle\right.$ In, Out, $Q$, out, $\left.E, q_{i}\right\rangle$ be a synchronous system and $p_{1}, q_{1} \in Q$ s.t. $p_{1} \nsim q_{1}$. A constructive proof of $p_{1} \nsim q_{1}$ is the data of (at least) two separating runs $r_{1} \in \operatorname{Runs}_{S}^{*}\left(p_{1}, w\right)$ and $r_{2} \in \operatorname{Runs}_{S}^{*}\left(q_{1}, w\right)$ :

$$
r_{1}=p_{1} \cdot a_{1} \cdot p_{2} \cdot a_{2} \ldots a_{n} \cdot p_{n} \text { and } r_{2}=q_{1} \cdot a_{2} \cdot q_{2} \cdot a_{2} \ldots a_{n} \cdot q_{n} \text {. }
$$

These runs are labelled on input by a finite word $w=a_{1} . a_{2} \ldots a_{n}$ called separator, and generate output words $o_{1} \in$ Out $^{*}=\operatorname{out}\left(p_{1}\right)$. out $\left(p_{2}\right) \ldots \operatorname{out}\left(p_{n}\right)$ and $o_{2} \in$ Out $^{*}=\operatorname{out}\left(q_{1}\right)$. out $\left(q_{2}\right) \ldots \operatorname{out}\left(q_{n}\right)$. More generally, any separator $w$ induces a nonempty set $\mathcal{O}\left(p_{1}, q_{1}, w\right)$ of pairs of different output words $\left(o_{1}, o_{2}\right)$ generated by separating runs s.t. $o_{1} \in \mathcal{L}_{S}^{*}\left(p_{1}, w\right), o_{2} \in \mathcal{L}_{S}^{*}\left(q_{1}, w\right)$.

A separator $w$ is deterministic when all its runs are separating, i.e. all runs stems from a proof of $p_{1} \nsim q_{1}$. The set of separators of two states $p, q$ is noted $\mathrm{S}(p, q)$, and the set of deterministic separators is noted $\mathrm{DS}(p, q)$. Note that $\mathrm{DS}(p, q) \subseteq \mathrm{S}(p, q)$.

Two states $p, q$ are said to be strongly separable, noted $p)(q$, iff all infinite inputs words are prefixed with a deterministic separator.

Fig. 2 shows two separable LTS. The fact that they are non-bisimilar is proved by the existence of two separators (although one would suffice) of length two and three, as emphasized by the dotted paths.

Once separability of two states defined, we can define what is an observable effect and when it occurs. This is based on observing the differences in the output word pairs generated by a separator.

Definition 6 (Observable effect). Let $p, q \in Q$ be two states. Let $w \in \operatorname{In}^{*}$ be an input word. The observable effects are generated by all the prefixes of $w$ wich are separators.

$$
\begin{aligned}
& \operatorname{diff}_{p, q}: \prod_{w \in I n^{*}} \mathbb{N}^{<|w|} \rightarrow \mathbb{1}+(\text { Out } \times \text { Out }) \\
& \operatorname{diff}_{p, q}(w, n)=\left(x_{1}, x_{2}\right) \leftrightarrow \\
& \quad \operatorname{prefix}(w, n+1) \in \mathrm{S}(p, q) \wedge\left(o_{1}, o_{2}\right) \in \mathcal{O}(p, q, \operatorname{prefix}(w, n+1)) \wedge
\end{aligned}
$$

$\operatorname{diff}_{p, q}(w, n)$ returns $\star \in \mathbb{1}$ if there is no difference at index $n$ or a pair $\left(o_{1}[n], o_{2}[n]\right)$ s.t. $o_{1}[n] \neq o_{2}[n]$ at the same index. These differences are the observable effects induced by $w$.

The existence of a separator ensures that there may be an observable effect. Fig. 3 show two systems (whose state space is quotiented by bisimulation equivalence) with an unknown output data $X$ in Fig. 


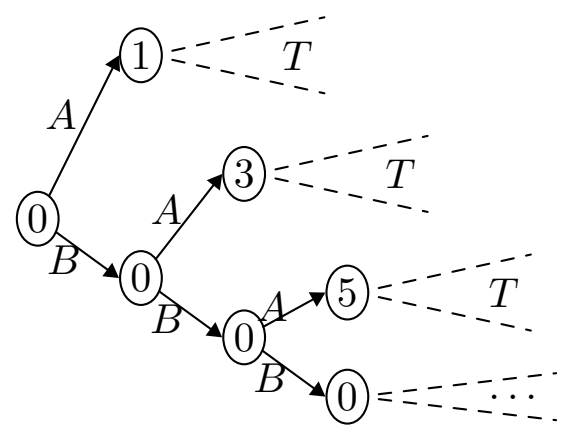

(a)

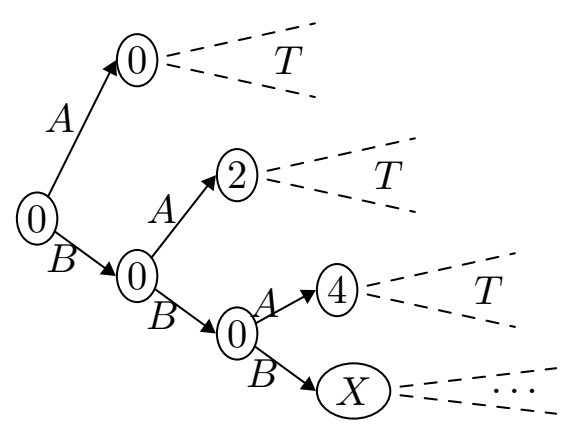

(b)

Figure 3: Partial separability

3(b). State names are omitted and the nodes only contain the output data. If $X=0$, the initial states are separable with the words of the language $B^{*} A$. These are deterministic separators: reading them on input ensures an observable effect. We observe that the input words of the language $B^{\infty}$ do not yield an observable effect. On the other hand, if $X \neq 0$, all infinite words are prefixed by a (deterministic) separator. An eventual observable reaction is guaranteed.

\subsection{Reaction time}

Since we work with logical time, the occurrence times of observable effects are their indices in the associated output traces. We may define the reaction time of a state as the maximum of the occurrence time of the first observable effect. This yields two possible views of reaction time: an optimistic one (an observable effect may arise ...) and a pessimistic one (an observable effect must arise). Moreover, the reaction time of a state can be valid for all contexts or just for some. Our application domain requires that we choose a pessimistic approach. Compositionality in turn requires that we quantify over all possible contexts when defining reaction time, as will be shown later.

Definition 7 (Deterministic reaction time). The (deterministic) reaction time of a state w.r.t. an input is the maximum number of transitions that must be performed to see the first observable effect arise, for any input. Let $q \in Q$ be a state s.t. reactive $(q)$ holds. We note by detreactime $(q)=t$ the fact that $q$ has a reaction time of t transitions, where:

$$
\begin{aligned}
\operatorname{detreactime}(q)=\max \{n \mid & \left.\left(a_{1}, a_{2}\right) \in \operatorname{DSepPairs}(q), q \stackrel{a_{1}}{\rightarrow} q_{1}, q \stackrel{a_{2}}{\rightarrow} q_{2}, q_{1}\right)\left(q_{2},\right. \\
& w \in \operatorname{In}^{\omega}, \operatorname{diff}_{q_{1}, q_{2}}(w, n) \neq \star \wedge \\
& \left.\forall n^{\prime}<n, \operatorname{diff}\left(w, n^{\prime}\right)=\star\right\}
\end{aligned}
$$

\section{Observable effects under composition}

In the previous section, we have defined a notion of observable effects for synchronous systems. In this section, we will investigate the way observable effects evolve when synchronous systems are composed. To this end, we will define a small process algebra, inspired by the category-theoretical work of Abramsky on concurrency [1]. 


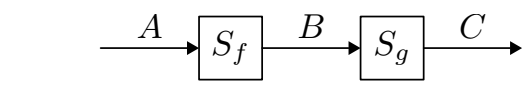

SEQ-NEXT

$\frac{q_{f} \stackrel{i n_{f}}{\longrightarrow} q_{f}^{\prime} \quad q_{g} \stackrel{\text { out }_{f}\left(q_{f}\right)}{\longrightarrow} q_{g}^{\prime}}{\left(q_{f}, q_{g}\right) \stackrel{i n_{f}}{\longrightarrow}\left(q_{f}^{\prime}, q_{g}^{\prime}\right)}$

SEQ-OUTPUT

out $_{g \circ f}\left(q_{f}, q_{g}\right)=$ out $_{g}\left(q_{g}\right)$

(a) Sequential composition

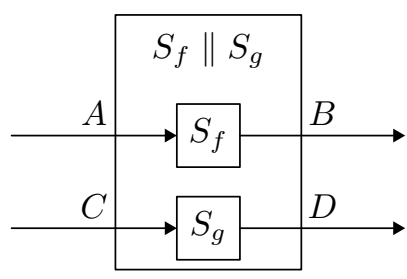

PAR-NEXT

$\frac{q_{f} \stackrel{i n_{f}}{\longrightarrow} q_{f}^{\prime} \quad q_{g} \stackrel{i n_{g}}{\longrightarrow} q_{g}^{\prime}}{q_{f}\left\|q_{g} \stackrel{\left\langle i n_{f}, i n_{g}\right\rangle}{\longrightarrow} q_{f}^{\prime}\right\| q_{g}^{\prime}}$

PAR-OUTPUT

$\overline{\text { out }_{f \| g}\left(q_{f} \| q_{g}\right)=\left\langle\operatorname{out}_{f}\left(q_{f}\right), \text { out }_{g}\left(q_{g}\right)\right\rangle}$

(b) Parallel composition

Figure 4: Definition of the composition operations

\subsection{Data types}

In order to model multiple input-output ports, we will force a monoidal structure on the data processed by our synchronous systems. Let Basic $=\{$ int $; \mathbf{b o o l} ; \mathbb{1} ; \ldots\}$ be a set of basic datatypes. The set of datatypes is the monoid $\langle\mathfrak{D}, \times\rangle$ generated by Basic and closed by cartesian product.

\subsection{Composition operators}

Our composition operators are sequential composition and parallel composition. The transition relations of the compound systems are defined in a classic way, using a small-step semantics given by SOS inference rules (c.f. Fig. 4).

Sequential composition. Let $A, B, C$ be three sets. Let $S_{f}=\left\langle A, B, Q_{f}, E_{f}\right.$, out ou $\left._{f}, q_{i, f}\right\rangle$ and $S_{g}=\langle B, C$, $Q_{g}, E_{g}$, out $\left.{ }_{g}, q_{i, g}\right\rangle$ be two systems. The sequential composition $S_{g} \circ S_{f}$ proceeds by redirecting the output of $S_{f}$ to the input of $S_{g}$. The compound system is $S_{g} \circ S_{f}=\left\langle A, C, Q_{f} \times Q_{g}, E_{g \circ f}\right.$, out $\left.{ }_{g \circ f},\left(q_{i, f}, q_{i, g}\right)\right\rangle$, where $E_{g \circ f}$ and out ${ }_{g \circ f}$ are defined in Fig. 4.

Parallel composition. Let $A, B, C, D$ be four sets. Let $S_{f}=\left\langle A, B, Q_{f}, E_{f}\right.$, out $\left.f, q_{i, f}\right\rangle$ and $S_{g}=\langle C$, $D, Q_{g}, E_{g}$, out $\left.{ }_{g}, q_{i, g}\right\rangle$ be two systems. The parallel composition proceeds by pairing the respective transitions of $S_{f}$ and $S_{g}$ in a synchronous way. The compound system is $S_{f} \| S_{g}=\left\langle A \times C, B \times D, Q_{f} \times\right.$ $Q_{g}, E_{f \| g}$, out $\left._{f \| g},\left(q_{i, f}, q_{i, g}\right)\right\rangle$ where $E_{f \| g}$ and out $f \| g$ are also defined in Fig. 4 .

Other operations. An other important operation is the feedback. We omit it for space reasons, but it must be noted that it exhibits the same behavior as sequential composition. The other operations necessary to make our definitions into an usable process algebra are structural ones, like data duplication, erasure, etc. These important details are omitted from the following study. 


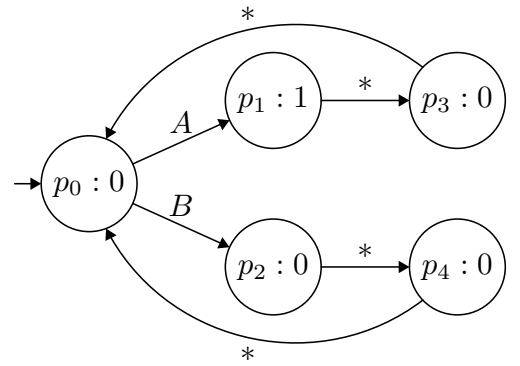

Machine $S_{f}$

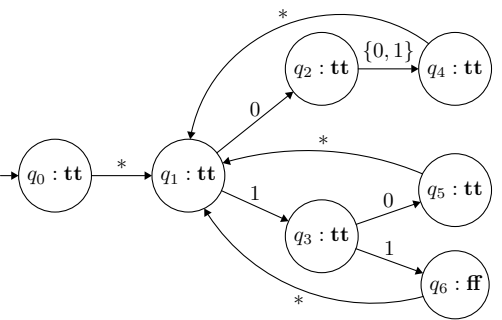

Machine $S_{g}$

Figure 5: Example of disappearing separator

\subsection{Observable effects w.r.t. sequential composition}

In this section, we study the behavior of the observable effects of systems when they are composed. We restrict our attention to sequential composition since it is easy to show that parallel composition doesn't alter the behavior of the sub-components. We show that under sequential composition, whenever reactivity still holds, the observable effects can vary arbitrarily. Our examples will be given on Moore machines whose state space is not quotiented by bisimulation equivalence.

The proof that reactivity can be lost follows the same argument that shows that the composition of two non-constant total functions can be constant. The figure to the right shows the composition of two reactive Moore machines $S_{f}$ and $S_{g}$ whose composition is not reactive. In this example, this stems from the fact that the observable effect $(0,1)$ of the input received in state $p_{0}$ of the machine $S_{f}$ is not "taken into account" by the machine $S_{g}$, i.e. $(0,1)$ is not a separating pair of state $q_{1}$.

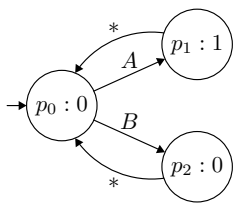

Machine $S_{f}$

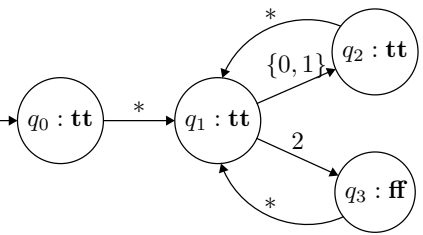

Machine $S_{g}$

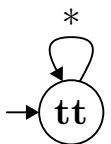

Machine $S_{g} \circ S_{f}$

The fact that an observable effect of $S_{f}$ is a separating pair of $S_{g}$ is not enough to guarantee an observable effect on output. Sequential composition restricts the input language of the system in receiving position (here, $S_{g}$ ). This means that separators can appear and disappear arbitrarily. The two Moore machines in Fig. 5 are modifications of the earlier ones. The states $p_{0}$ and $q_{1}$ are still reactive, but when composed the output symbols on $p_{3}$ and $p_{4}$ restricts the set of inputs of the states $q_{2}$ and $q_{3}$ to the word 0 . Thus, $q_{2}$ and $q_{3}$ are no more separable and the result is the constant machine shown earlier.

The conclusion of this study confirms the intuition: there is no general way of guaranteeing functional dependencies. These results extend to reaction time, which is not conserved: the receiving machine may ignore the first observable effect and take into account ulterior ones.

In verification terms, this means that in order to verify that the composition of two systems is reactive, a full search of the state space for separators must be undertaken. In the next section, an approximate but compositional method to simplify this process is proposed. 


\section{Under-approximating observable effects}

This section proposes a partial solution to some problems encountered earlier, namely:

1. the fact that non-deterministic separators do not guarantee an observable effect,

2. the non-compositionality of reactivity and observable effects.

We proceed by reducing our focus to the cases where reactivity, which is a branching-time property of states, can be reduced to a linear-time one. We show how to compute the separators and separating pairs which are preserved when "merging" all branches of the computation tree.

Let us assume the existence of two sets of data In and Out. Let $q$ be a state such that reactive $(q)$ holds, and let $\left(a_{1}, a_{2}\right) \in \operatorname{SepPairs}(s)$ be a separating pair of inputs. In Sec. 4.3 , we observed that in order to ensure the occurrence of an observable effect and the existence of a reaction time, $q$ must be such that all inputs are deterministic separators for all $q_{1}$ and $q_{2}$ s.t. $q \stackrel{a_{1}}{\rightarrow} q_{1}$ and $q \stackrel{a_{2}}{\rightarrow} q_{2}$. If this condition is met, we can compute deterministic observable effects, i.e. effects which exists for all separators. Similarly, we can define deterministic separating pairs.

First, we define some operations in order to merge sequences of observable effects. We define the operation $\oplus:(\mathbb{1}+$ Out $\times$ Out $) \times(\mathbb{1}+$ Out $\times$ Out $) \rightarrow(\mathbb{1}+$ Out $\times$ Out $)$ as:

$$
\begin{aligned}
& x \oplus x=x \\
& x \oplus y=\star \text { if } x \neq y .
\end{aligned}
$$

The extension of this operation to sequences of symbols on $(\mathbb{1}+O u t \times O u t)$ is defined straightforwardly. If $d_{1}, d_{2} \in(\mathbb{1}+O u t \times O u t)^{\omega}$ are two infinite sequences, their merging is also noted $d_{1} \oplus d_{2}$.

Definition 8 (Deterministic observable effects, observational order). Let $q$ be a state s.t. reactive $(q)$ holds. The sequence of deterministic observable effects of $q$ is noted $\operatorname{DOE}(q)$ and is defined as follows:

$$
\operatorname{DOE}(q)=\underset{\left(a_{1}, a_{2}\right) \in \operatorname{SepPairs}(q)}{\bigoplus}\left\{\operatorname{diff}_{q_{1}, q_{2}}(w) \mid q \stackrel{a_{1}}{\rightarrow} q_{1}, q \stackrel{a_{2}}{\rightarrow} q_{2}, w \in \operatorname{In}^{\omega}\right\} .
$$

It is possible to define a relation $<\subseteq(\mathbb{1}+\text { Out } \times \text { Out })^{\omega} \times(\mathbb{1}+\text { Out } \times \text { Out })^{\omega}$, where:

$$
w_{1} \prec w_{2} \leftrightarrow \exists ! i,\left(\forall j \neq i, w_{1}[j]=w_{2}[j]\right) \wedge\left(w_{1}[i]=\star \wedge w_{2}[i] \neq \star\right) .
$$

The reflexive-transitive closure of $<$ is the observational order and is noted $\leqslant$. The set $\operatorname{ObsOrder}(q)$ of infinite strings partially ordered by $\leqslant$ which has $\operatorname{DOE}(q)$ as greatest element and $\star^{\omega}$ as least element is called by extension the observational order on $q$.

We must also define linear time-proof separating pairs, called strongly separating pairs. Let's consider the systems in Fig. 6, in which only the output data is displayed and state names are omitted. The systems 1 and 2 are symmetrical and have both $(A, B)$ as a separating pair for their initial state. However, $(A, B)$ is not a separating pair for the union of the two systems. We must define a notion of separating pair for two systems which resists their union.

Definition 9 (Strongly separating pairs). Let $q_{1}, q_{2}$ be two states s.t. reactive $\left(q_{1,2}\right)$ holds. The pair $\left(a_{1}, a_{2}\right) \in \operatorname{SepPairs}\left(q_{1,2}\right)$ is a strongly separating pair of the union of $q_{1}$ and $q_{2}$ if and only if:

$$
\left(\exists q_{1} \stackrel{a_{1}}{\rightarrow} q_{1}^{\prime}, \forall q_{2} \stackrel{a_{2}}{\rightarrow} q_{2}^{\prime}, q_{1}^{\prime} \nsim q_{2}^{\prime}\right) \vee\left(\exists q_{1} \stackrel{a_{2}}{\rightarrow} q_{1}^{\prime}, \forall q_{2} \stackrel{a_{1}}{\rightarrow} q_{2}^{\prime}, q_{1}^{\prime} \nsim q_{2}^{\prime}\right) .
$$




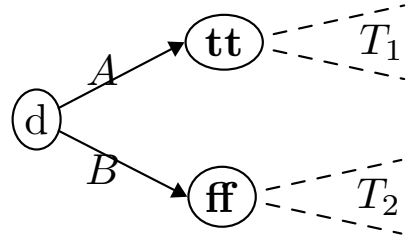

(a) System 1

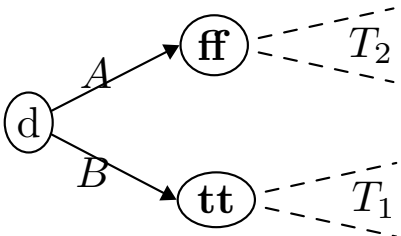

(b) System 2

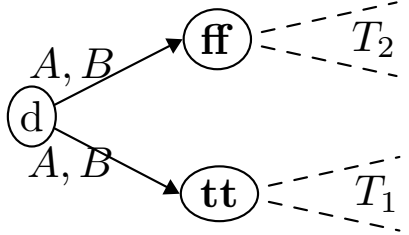

(c) Union of systems 1 and 2

Figure 6: Union of transition systems

The set of strongly separating pairs of $q_{1}$ and $q_{2}$ is noted $\operatorname{SSP}\left(q_{1}, q_{2}\right)$. Note that $\operatorname{SepPairs}(q)=\operatorname{SSP}(q, q)$. The sequence of strong separating pairs of $q_{1}$ and $q_{2}$ is noted SSPseq $\left(q_{1}, q_{2}\right)$ and is defined as follows:

$$
\operatorname{SSPseq}\left(q_{1}, q_{2}\right)=\operatorname{SSP}\left(q_{1}, q_{2}\right) \cdot\left(\bar{\bigcap}\left\{\operatorname{SSPseq}\left(q_{1}^{\prime}, q_{2}^{\prime}\right) \mid\left(a_{1}, a_{2}\right) \in \operatorname{SepPairs}(q), q_{1} \stackrel{a_{1}}{\rightarrow} q_{1}^{\prime}, q_{2} \stackrel{a_{2}}{\rightarrow} q_{2}^{\prime}\right\}\right),
$$

where $\bar{\cap}$ is the extension of set intersection to sequences of sets. Now, let $q$ be s.t. reactive $(q)$ holds. The sequence of strongly separating pairs of $q$ is:

$$
\operatorname{SSPseq}(q)=\operatorname{SepPairs}(q) \cdot \bar{\bigcap}\left\{\operatorname{SSPseq}\left(q_{1}, q_{2}\right) \mid\left(a_{1}, a_{2}\right) \in \operatorname{SepPairs}(q), q \stackrel{a_{1}}{\rightarrow} q_{1}, q \stackrel{a_{2}}{\rightarrow} q_{2}\right\} .
$$

Deterministic observable effects are in fact an abstraction of the original system. The concretization operation is the function associating to a sequence of deterministic observable effects the set of all systems which have at least these deterministic observable effects (w.r.t. $\leqslant$ ). Using this abstraction, checking the compositionality of sequential composition is straightforward.

Lemma 1 (Sequential composition of deterministic observable effects ensures reactivity). Let $S_{f}=\langle A, B$, $Q_{f}, E_{f}$, out $\left.f, q_{i, f}\right\rangle$ and $S_{g}=\left\langle B, C, Q_{g}, E_{g}\right.$, out $\left.{ }_{g}, q_{i, g}\right\rangle$ be two systems. Let $q_{f} \in Q_{f}$ and $q_{g} \in Q_{g}$ be two states such that $\left(q_{f}, q_{g}\right)$ is in the state space of the sequential composition $S_{g} \circ S_{f}$. We have reactive $\left(q_{f}, q_{g}\right)$ if:

$$
\exists d \in \operatorname{ObsOrder}\left(q_{f}\right), \exists i, d[i] \in \operatorname{SSPseq}\left(q_{g}\right)[i+1] .
$$

Proof. Let $i \in \mathbb{N}$ be such that $d[i] \in \operatorname{SSPseq}\left(q_{g}\right)[i+1]$. Having $d[i]=\left(x_{1}, x_{2}\right)$ implies that reactive $\left(q_{f}\right)$ holds. Hence, there exists $a_{1} \neq a_{2}$ s.t. $\exists q_{f} \stackrel{a_{1}}{\rightarrow} q_{f}^{1}, \forall q_{f} \stackrel{a_{2}}{\rightarrow} q_{f}^{2}, q_{f}^{1} \nsim q_{f}^{2}$. By hypothesis, we know that all input words are separators of all such $\left(q_{f}^{1}, q_{f}^{2}\right)$. By definition, $d[i]$ is an observable effect of all these separators. Hence, for all input words $w$ there will exist two runs $q_{f}^{1} \stackrel{\text { prefix }(w, i)}{\longrightarrow} r_{f}^{1}$ and $q_{f}^{2} \stackrel{\operatorname{prefix}(w, i)}{\longrightarrow} r_{f}^{2}$ s.t. out $\left(r_{f}^{1}\right)=$ $x_{1}$ and out $\left(r_{f}^{2}\right)=x_{2}$. By definition of the sequential composition, this induces the runs $\left(q_{f}^{1}, q_{g}^{1}\right) \stackrel{\text { prefix }(w, i)}{\longrightarrow}$ $\left(r_{f}^{1}, r_{g}^{1}\right)$ and $\left(q_{f}^{2}, q_{g}^{1}\right) \stackrel{\operatorname{prefix}(w, i)}{\longrightarrow}\left(r_{f}^{2}, r_{g}^{2}\right)$ (with $\left.q_{g} \stackrel{\text { out }(f)}{\longrightarrow} q_{g}^{1}\right)$. Since $\left(x_{1}, x_{2}\right) \in \operatorname{SSP}\left(r_{g}^{1,2}\right)$ (by definition of SSPseq $),\left(q_{f}^{1}, q_{g}^{1}\right) \nsim\left(q_{f}^{2}, q_{g}^{1}\right)$.

The compositionality of this approach stems from the fact that for any systems $S_{f}, S_{g}$ and respective states $q_{f}$ and $q_{g}$, an element of $\operatorname{ObsOrder}\left(q_{f}, q_{g}\right)$ can be computed using other elements from ObsOrder and SSP.

Definition 10 (Compositionality of deterministic observable effects). Let $q_{f} \in Q_{f}, q_{g} \in Q_{g}$ be two states. Let doe $f \in \operatorname{ObsOrder}\left(q_{f}\right)$ and $d s p_{g} \in \operatorname{SSP}\left(q_{g}\right)$. If there exists a $t$ s.t. doe $f[t] \in d s p_{g}[t+1]$ then there exists an element doe $t_{g \circ f}^{t} \in \operatorname{ObsOrder}\left(q_{f}, q_{g}\right)$ s.t.:

$$
d o e_{g \circ f}^{t}=\star \star_{\star} \star^{t} . \bigoplus\left\{\text { doe }_{g^{\prime}} \mid\left(q_{f}, q_{g}\right) \rightarrow^{t+1}\left(q_{f}^{\prime}, q_{g}^{\prime}\right), \text { doe }_{g^{\prime}} \in \operatorname{Obs} \operatorname{Order}\left(q_{g}^{\prime}\right)\right\}
$$




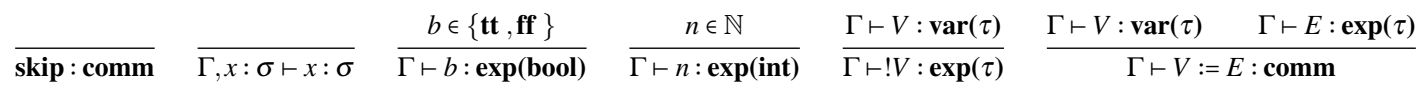

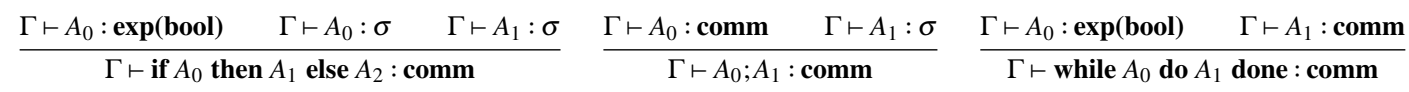

$$
\begin{aligned}
& \frac{\Gamma \vdash A_{0}: \pi_{0} \text { Out } \quad \ldots \quad \Gamma \vdash A_{\mid \text {Out } \mid-1}: \pi_{\mid \text {out } \mid-1} \text { Out }}{\Gamma \vdash \operatorname{tick}\left(A_{0} \ldots A_{\mid \text {Out } \mid-1}\right): \mathbf{c o m m}} \quad \frac{}{\Gamma \vdash \text { get }_{i}: \pi_{i} \text { In } \quad i \in \mathbb{N}^{<|I n|}}
\end{aligned}
$$

Figure 7: Definition of well-typed programs.

This element is computed by merging the deterministic observable effects of states of $q_{g}$ reachable in $t+1$ transitions. The initial $\star$ stems from the delay induced by communication in the synchronous model.

A similar property holds for separating pairs. We only describe informally how to proceed, since the general idea is similar to the case of deterministic observable effects. A strongly separating pair of $q_{f}$ exists in $S_{g} \circ S_{f}$ if the states $q_{f}^{1}, q_{f}^{2}$ reachable by this pair have a common observable effect (computable using $\oplus$ ) which corresponds to a strongly separating pair of $q_{g}$.

\section{Example}

As explained in the introduction, our work focuses on a real-time system called OASIS, which provides a real-time kernel and a multi-agent synchronous-like language called PsyC (an extension of C with synchronous primitives). We have given a formal semantics of a simplification of PsyC called PsyALGOL. We will use this semantics to highlight a common use case of our framework.

\subsection{Syntax and semantics of a simple synchronous language.}

In order to give the semantics of a program, we have to define how its LTS is generated. We briefly survey a subset of the syntax of the language. The connection between the semantics and the resulting LTS should be straightforward, we will thus omit the derivations.

\section{Syntax.}

The syntax definition is given in an inductive way using inference rules on judgments of the shape $\Gamma \vdash M: \sigma$, meaning "in the context $\Gamma$, the program $M$ has type $\sigma$ ". A context is a list of the shape $x_{1}: \sigma_{1}, \ldots, x_{n}: \sigma_{k}$. It associates variables $x_{i}$ to their types $\sigma_{i}$. For the sake of simplicity, we assume that all variables are declared beforehand and initialized to their default value. We ignore procedures and we keep the other syntactical forms as simple as possible. The types $\sigma$ and default values are defined as follows:

$$
\begin{aligned}
& \tau::=\text { int } \mid \text { bool } \quad \text { defaul }_{\text {int }}=0 \\
& \sigma::=\operatorname{comm}|\operatorname{var}(\tau)| \exp (\tau) \quad \text { defaul }_{\text {bool }}=\mathbf{f f}
\end{aligned}
$$

Assuming that the programs have input and output types $\langle$ In, Out $\rangle$, the set Prog of correctly typed programs is defined in Fig. 7. We omit the arithmetical operators. 


\section{Semantics.}

We will define the operational semantics for our language as a small-step relation. An operational semantics is usually a kind of relation associating a program in its initial configuration to its final outcome (be it a final value or divergence).

Our aim is slightly different: we want to view the evaluation of a program as a synchronous system. This means that instead of producing a final value or diverging, we want to quantify over all possible inputs at each logical step, and produce a LTS. In order to simplify matters, our LTS will be given in unfolded form, as an infinitely deep tree. Each step of the evaluation will grow this tree downward, and the limit of this process will be the semantics of the program. Let's proceed to some definitions.

Definition 11. The set of configurations is Config $\triangleq$ Store $(V) \times I n \times$ Prog, where $V$ is the set of variables of the program and Store $(V)$ is a mapping from variables to constants.

Definition 12. The sets Trees of finite (resp. infinite) partial evaluation trees are generated by the inductive (resp. co-inductive) interpretation of the following rules.

$$
\begin{aligned}
& \text { LEAF NODE } \\
& \frac{\text { conf } \in \text { Config }}{\text { conf } \in \text { Trees }} \quad \frac{\text { out } \in \text { Out } \quad \forall i n, t r_{\text {in }} \in \text { Trees }}{\left(\text { out },\left\{\left(\text { in }, t r_{i n}\right) \mid i n \in I n\right\}\right)}
\end{aligned}
$$

Let $T$ be a partial evaluation tree with leaves $\left(s_{i}, i_{i}, \operatorname{prog}_{i}\right)$. Given a syntactical mapping map: Prog $\rightarrow$ Prog, we note map $\downarrow T$ the extension of map to the leaves of $T$ such that ( $\operatorname{map} \downarrow T$ ) has leaves $\left(s t_{i}, i n_{i}, \operatorname{map}\left(\operatorname{prog}_{i}\right)\right)$.

The one-step reduction relation $\rightarrow \subseteq$ FiniteTrees $\times$ FiniteTrees is defined in terms of a relation $\rightarrow \subseteq$ Config $\times$ FiniteTrees defined by the rules in Fig. 8 .

We define $\rightarrow$ as the application of $\rightarrow$ to the leaves of a tree. From there, we can define the standard reflexive-transitive closure of $\rightarrow$ and its co-inductive counterpart as in [9].

\subsection{Example of synchronous programs.}

We will study the behavior of two programs, whose texts and associated (deterministic) LTS are displayed in Fig. 9. The first program captures an input data at the beginning of the outer loop and releases it on output when the inner loop finishes executing itself. The second program proceeds similarly, except that the inner loop termination is ensured by the usage of a decreasing counter $y$ initialized to a constant $N$. In the LTS of program 2, this corresponds to the dashed transition between $q_{1}$ and $q_{2}$, which should be understood as $N-2$ omitted states with decreasing values of $y$.

We want to check whether the data inputted at the tick $(! x)$; lines highlighted in both programs yield a finite reaction time. These instructions corresponds to states $p_{0}, p_{2}$ in LTS 1 , and $q_{0}, q_{3}$ in LTS 2 . Thus, in order for these instructions to be "reactive", the corresponding states must have a finite reaction time. In order to study this, we will compute their deterministic observable effects.

Program 1. The set of separating pairs of $p_{0}$ and $p_{2}$ is $\operatorname{SepPairs}\left(p_{0,2}\right)=\{(\mathbf{t t}, \mathbf{f f})\}$. This fact is proved by the transitions $p_{0} \stackrel{\mathbf{t t}}{\longrightarrow} p_{1}, p_{0} \stackrel{\mathbf{f f}}{\longrightarrow} p_{0}$, and $p_{2} \stackrel{\mathbf{t t}}{\longrightarrow} p_{1}, p_{2} \stackrel{\mathbf{f f}}{\longrightarrow} p_{0}$ where $p_{0} \nsim p_{1}$. The only separator of $\left(p_{0}, p_{1}\right)$ is the one-symbol word $w=\mathbf{f f}$. This fact is proved by the transitions $p_{0} \stackrel{\text { ff }}{\longrightarrow} p_{0}$ and $p_{1} \stackrel{\text { ff }}{\longrightarrow} p_{2}$, where out $\left(p_{0}\right) \neq \operatorname{out}\left(p_{2}\right)$. The separator $w$ is deterministic, since the underlying automaton is itself deterministic. This separator induces a pair of output words $\left(o_{1}, o_{2}\right)=(\mathbf{f f} . \mathbf{f f}, \mathbf{f f}$.tt $)$ and thus an observable 

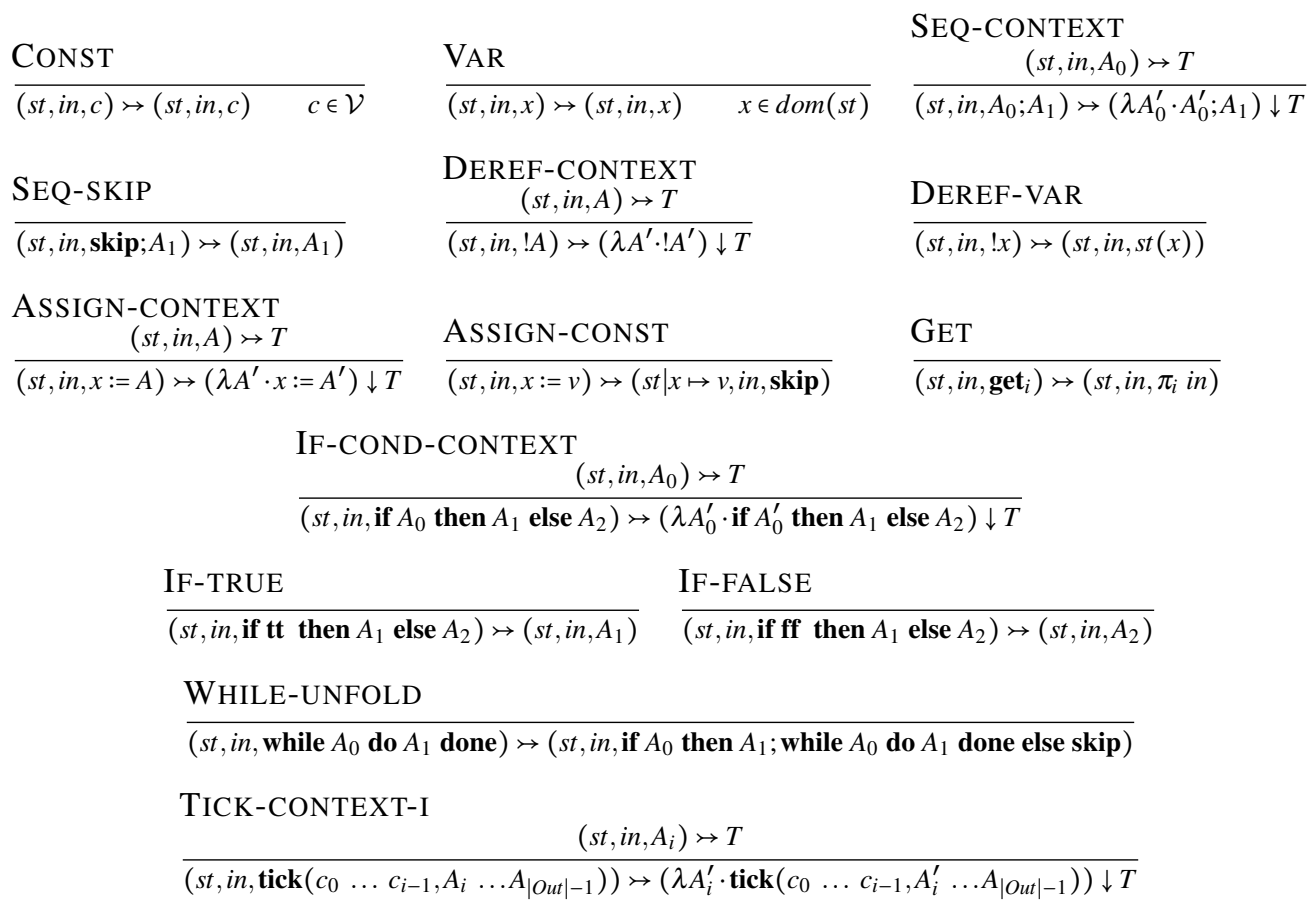

TICK-CONSTANT

$\overline{\left(s t, \text { in }, \operatorname{tick}\left(c_{0} \ldots c_{|O u t|-1}\right)\right) \nrightarrow\left(\left(c_{0} \ldots c_{|O u t|-1}\right),\{(\text { input },(\text { st }, \text { input }, \text { skip })) \mid \text { input } \in \text { In }\}\right)}$

Figure 8: One-step reduction relation

effect diff $p_{p_{0}, p_{1}}(w, 1)=(\mathbf{f f}, \mathbf{t t})$. However, this observable effect is not deterministic, since there exist an infinite input word tt ${ }^{\omega}$ which generates no observable effect. Thus, the sequence of observable effects of $p_{0}$ and $p_{2}$ is $\star^{\omega}$ and the reaction time for the highlighted line instruction does not exist (or, equivalently, is infinite).

Program 2. The set of separating pairs of $q_{0}$ and $q_{3}$ is still $\operatorname{SepPairs}\left(q_{0,3}\right)=\{(\mathbf{t t}, \mathbf{f f})\}$. The corresponding transitions are $q_{0} \stackrel{\text { tt }}{\longrightarrow} q_{1}, q_{0} \stackrel{\text { ff }}{\longrightarrow} q_{0}$ and $q_{3} \stackrel{\text { tt }}{\longrightarrow} q_{1}, q_{3} \stackrel{\text { ff }}{\longrightarrow} q_{0}$ with $q_{0} \nsim q_{1}$. The (deterministic) separators of $\left(q_{0}, q_{1}\right)$ are $\mathrm{S}\left(q_{0}, q_{1}\right)=\mathrm{DS}\left(q_{0}, q_{1}\right)=\left\{\mathbf{f f} ; \mathbf{t t} . \mathbf{f f} ; \mathbf{t t} . \mathbf{t t} . \mathbf{f f} ; \ldots ; \mathbf{t t}^{N-2}\right.$.ff; $\mathbf{t t}^{N-1}$.bool $\}$. The table below lists the observable differences associated to each separator.

$$
\begin{aligned}
& \text { ff } \quad \mapsto \quad \star .(\mathbf{f f}, \mathbf{t t}) . \star^{\omega} \\
& \text { tt.ff } \quad \mapsto \quad \star . \star .(\text { ff }, \text { tt }) .{ }_{\star} \omega \\
& \left.\mathbf{t t}^{N-2} \text {.ff } \quad \mapsto \quad \star^{N-1} \text {.(ff }, \mathbf{t t}\right) . \star^{\omega} \\
& \left.\mathbf{t t}^{N-1} \text {.bool } \mapsto \quad \star^{N} \text {.(ff }, \mathbf{t t}\right) . \star^{\omega}
\end{aligned}
$$

When merging these observable differences, we obtain $\operatorname{DOE}\left(q_{0,3}\right)=\star^{\omega}$. This means that even though the program 2 is reactive with a finite reaction time, it is still non-compositional within our simple framework. This is due to the fact that the observable effects occurrence time are non-uniform w.r.t. inputs, i.e. non-constant. 
PROGRAM 2

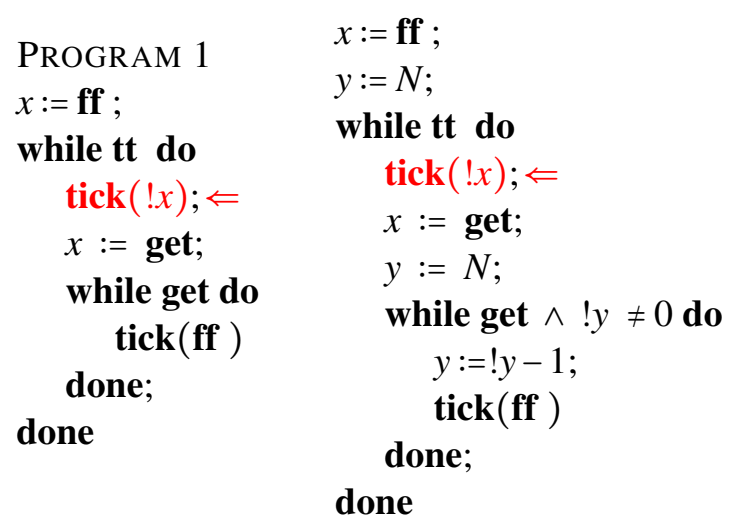

(a) Programs 1 and 2

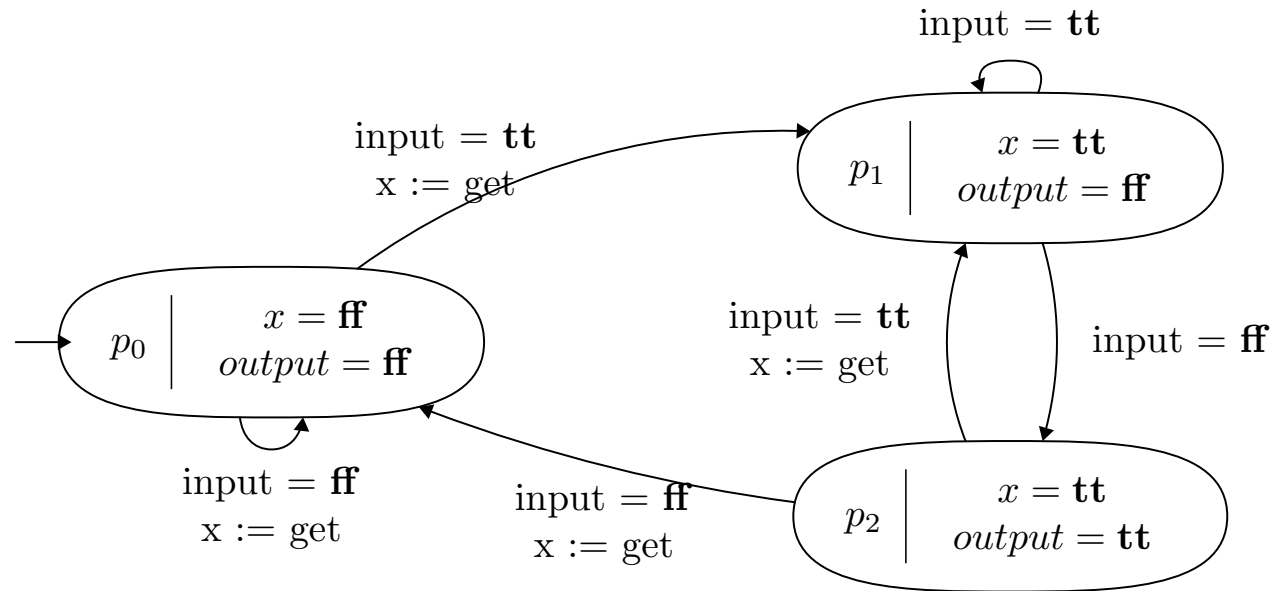

(b) LTS of program 1

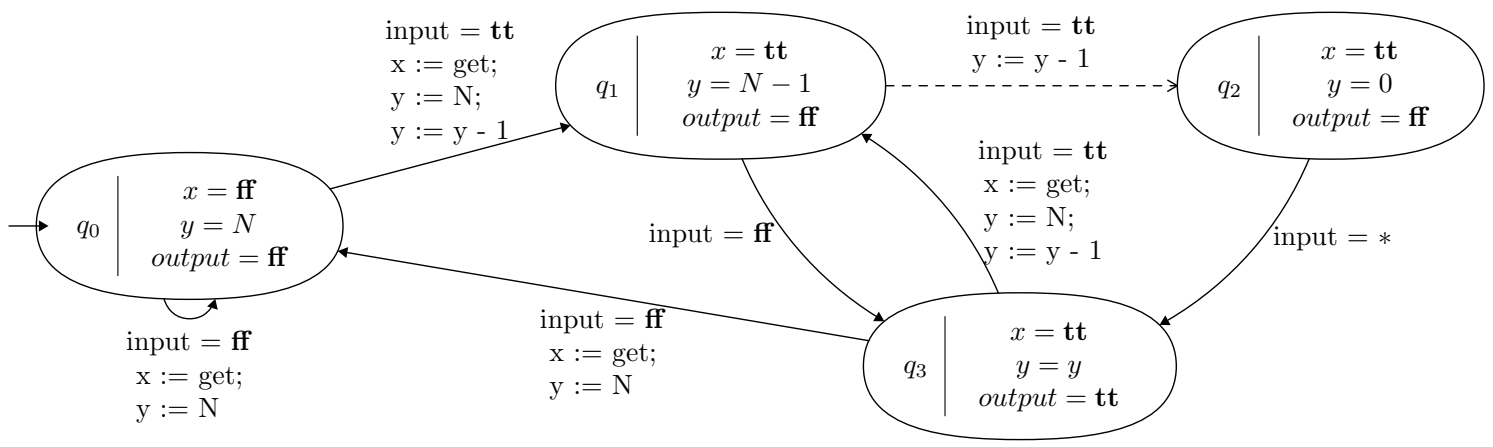

(c) LTS of program 2

Figure 9: LTS of programs 1 and 2 


\section{Conclusions and future works}

We have formalized in this paper the notions of functional dependency and reaction time for some synchronous systems. These notion are adapted to the formal investigation of reaction time constraints for the aforementioned synchronous systems. Functional dependencies were shown to be brittle and not suited to composition and verification. To answer this problem, we proposed an approximated method which gains compositionality by restricting its scope to deterministic separators.

Our work opens some other research directions: a broader investigation of the notion of reaction time in a more general setting [7] could prove fruitful and lead to simpler, more abstract and general definitions. Our composition operators are quite restricted, as shown in the example, but making it more flexible should be possible by making deterministic effects a function of some arbitrary decidable specification. It seems also possible to apply our ideas to the refinement-based development of systems.

We provide a formal framework allowing to reason on functional dependencies and reaction time which is amenable to automated verification. This effort should help the software designer and programmer to deliver reliable, predictable and efficient systems.

\section{References}

[1] S. Abramsky, S. Gay \& R. Nagarajan (1996): Interaction Categories and the Foundations of Typed Concurrent Programming. In M. Broy, editor: Proceedings of the 1994 Marktoberdorf Summer Sxhool on Deductive Program Design, Springer-Verlag, pp. 35-113.

[2] Rajeev Alur \& David L. Dill (1994): A Theory of Timed Automata. Theoretical Computer Science 126, pp. 183-235, doi:10.1016/0304-3975(94)90010-8

[3] R. Barbuti, C. Bernardeschi \& N. De Francesco (2002): Abstract interpretation of operational semantics for secure information flow. Inf. Process. Lett. 83(2).

[4] E. Clarke, D. Long \& K. McMillan (1989): Compositional model checking. In: Proceedings of the Fourth Annual Symposium on Logic in computer science, IEEE Press, Piscataway, NJ, USA, pp. 353-362, doi $10.1109 /$ LICS.1989.39190

[5] Vincent David, Jean Delcoigne, Evelyne Leret, Alain Ourghanlian, Philippe Hilsenkopf \& Philippe Paris (1998): Safety Properties Ensured by the OASIS Model for Safety Critical Real-Time Systems. In: SAFECOMP.

[6] E. Allen Emerson \& Joseph Y. Halpern (1982): Decision procedures and expressiveness in the temporal logic of branching time. In: Proceedings of the fourteenth annual ACM symposium on Theory of computing, STOC '82, ACM, New York, NY, USA, pp. 169-180, doi:10.1145/800070.802190

[7] Esfandiar Haghverdi, Paulo Tabuada \& George J. Pappas (2005): Bisimulation relations for dynamical, control, and hybrid systems. Theor. Comput. Sci. 342, pp. 229-261, doi $10.1016 /$ j.tcs.2005.03.045

[8] N. Halbwachs, P. Caspi, P. Raymond \& D. Pilaud (1991): The synchronous dataflow programming language Lustre. Proceedings of the IEEE 79(9), pp. 1305-1320, doi $10.1109 / 5.97300$.

[9] Xavier Leroy \& Hervé Grall (2008): Coinductive big-step operational semantics Available at http:// arxiv.org/abs/0808.0586.

[10] Edward F. Moore (1956): Gedanken Experiments on Sequential Machines. In: Automata Studies, Princeton U., pp. 129-153.

[11] Mike Stannett (2006): Simulation testing of automata. Formal Aspects of Computing 18, pp. 31-41. 10.1007/s00165-005-0080-y. 